\title{
String confinement in two-form lattice gauge theory
}

\author{
Tomoya Hayata $\odot^{1}$ and Arata Yamamoto ${ }^{2}$ \\ ${ }^{1}$ Nishina Center, RIKEN, Wako 351-0198, Japan \\ ${ }^{2}$ Department of Physics, The University of Tokyo, Tokyo 113-0031, Japan
}

(Received 3 September 2019; published 15 October 2019)

\begin{abstract}
We study the confinement between vortex strings in the lattice gauge theory of the dual Abelian Higgs model. The dual lattice gauge theory is described by a two-form gauge field coupled with a one-form gauge field. We calculate the string-antistring potential from the surface operator of the two-form gauge field. The linear confining potential appears in the string confinement phase, and it disappears in the string deconfinement phase. The phase diagram of the theory is also obtained.
\end{abstract}

DOI: 10.1103/PhysRevD.100.074504

\section{INTRODUCTION}

A quantum vortex string is a one-dimensional topological soliton. The existence of vortex strings was experimentally confirmed in superconductors [1] and superfluids [2]. It is also believed to exist in compact stars [3] and the Universe [4]. The circulation around a vortex string is quantized due to the single valuedness of a field variable. The quantized circulation is topologically protected, and thus, the vortex string is stable. The stability ensures the description as quasiparticles, e.g., interaction and dynamics of vortex strings.

The field theory with vortex strings is a dual to antisymmetric rank-2 tensor, i.e., two-form, gauge theory. The world sheets of vortex strings are described by the surface operator of a two-form gauge field. This is an analog of the Wilson loop operator in one-form gauge theory. The Wilson loop operator corresponds to the world lines of charged particles. The expectation value of the Wilson loop operator tells us the interaction, e.g., the confinement, between the particles. Similarly, the interaction between the vortex strings can be investigated from the surface operator of the two-form gauge field.

The two-form gauge theory can be nonperturbatively formulated by lattice regularization [5-10]. This is a higher-form generalization of the conventional lattice gauge theory, i.e., the lattice regularization of one-form gauge theory. The higher-form lattice gauge theory is sometimes called lattice gerbe theory $[11,12]$. The twoform lattice gauge theory enables us to study nonperturbative properties of a vortex string from first principles.

Published by the American Physical Society under the terms of the Creative Commons Attribution 4.0 International license. Further distribution of this work must maintain attribution to the author(s) and the published article's title, journal citation, and DOI. Funded by SCOAP ${ }^{3}$.
Although the vortex string is frequently studied in semiclassical analysis, it misses quantum fluctuation. Firstprinciples analysis is necessary to take into account quantum fluctuation, e.g., percolation [13-16] and superposition $[17,18]$. Such an analysis is particularly important near phase transitions or in finite volumes, where quantum fluctuation is non-negligible.

In this work, we study the confinement phenomenon in two-form lattice gauge theory. In Sec. II, we review the lattice formulation of two-form gauge theory. We consider the two-form gauge theory coupled with a one-form gauge field. This theory is dual to the Abelian Higgs model in the continuum limit [19]. The theory exhibits the confinement-deconfinement phase transition of vortex strings. We confirm this based on two analyses. In Sec. III, we define the Wilson surface operator and calculate the potential between a string and antistring. In Sec. IV, we draw the phase diagram of this theory by calculating a susceptibility.

\section{ABELIAN TWO-FORM LATTICE GAUGE THEORY}

We consider a novel lattice gauge theory in fourdimensional Euclid spacetime. The theory contains two kinds of Abelian gauge fields: the one-form gauge field $A_{\mu}(x)$ and the two-form gauge field $B_{\mu \nu}(x)$. The one-form gauge field is defined as a link variable between $x$ and $x+\hat{\mu}$,

$$
U_{\mu}(x)=e^{i a A_{\mu}(x)} .
$$

Here, $x$ denotes a site in a hypercubic lattice, $\hat{\mu}$ denotes the unit vector along the $\mu$ direction, and $a$ is lattice spacing. The two-form gauge field is defined as a plaquette variable whose vertices are at $x, x+\hat{\mu}, x+\hat{\nu}$, and $x+\hat{\mu}+\hat{\nu}$,

$$
\Gamma_{\mu \nu}(x)=e^{i a^{2} B_{\mu \nu}(x)}
$$


These variables are $\mathrm{U}(1)$ elements. The lattice action is constructed from these variables as

$$
\begin{aligned}
S_{\text {lat }}= & \kappa \sum_{x, \mu<\nu}\left(1-\frac{1}{2}\left(\tilde{U}_{\mu \nu}(x)+\tilde{U}_{\mu \nu}^{\dagger}(x)\right)\right) \\
& +\beta \sum_{x, \mu<\nu<\lambda}\left(1-\frac{1}{2}\left(\Gamma_{\mu \nu \lambda}(x)+\Gamma_{\mu \nu \lambda}^{\dagger}(x)\right)\right),
\end{aligned}
$$

with

$$
\begin{aligned}
U_{\mu \nu}(x)= & U_{\nu}^{\dagger}(x) U_{\mu}^{\dagger}(x+\hat{\nu}) U_{\nu}(x+\hat{\mu}) U_{\mu}(x), \\
& \tilde{U}_{\mu \nu}(x)=U_{\mu \nu}(x) \Gamma_{\mu \nu}(x), \\
\Gamma_{\mu \nu \lambda}(x)= & \Gamma_{\lambda \mu}^{\dagger}(x+\hat{\nu}) \Gamma_{\nu \lambda}^{\dagger}(x+\hat{\mu}) \Gamma_{\mu \nu}^{\dagger}(x+\hat{\lambda}) \\
& \times \Gamma_{\lambda \mu}(x) \Gamma_{\nu \lambda}(x) \Gamma_{\mu \nu}(x) .
\end{aligned}
$$

Here, $\kappa$ and $\beta$ are dimensionless coupling constants. In the continuum limit, this lattice gauge theory is dual to the Abelian Higgs model [19].

This theory has two kinds of local gauge symmetry. The one-form gauge transformation is defined with a unitary matrix $e^{i \theta(x)}$ as

$$
U_{\mu}(x) \rightarrow e^{i \theta(x+\hat{\mu})} U_{\mu}(x) e^{-i \theta(x)} .
$$

The minimal gauge-invariant observable is the plaquette operator $U_{\mu \nu}$. Since the action is written only by $U_{\mu \nu}$, it is manifestly invariant under the one-form gauge transformation. The two-form gauge transformation is defined with a unitary matrix $e^{i \lambda_{\mu}(x)}$ as

$$
\Gamma_{\mu \nu}(x) \rightarrow e^{i \lambda_{\nu}(x)} e^{i \lambda_{\mu}(x+\hat{\nu})} \Gamma_{\mu \nu}(x) e^{-i \lambda_{\nu}(x+\hat{\mu})} e^{-i \lambda_{\mu}(x)}
$$

and simultaneously

$$
U_{\mu}(x) \rightarrow e^{i \lambda_{\mu}(x)} U_{\mu}(x) .
$$

The minimal gauge-invariant observable is the plaquette operator $\tilde{U}_{\mu \nu}$ and the unit cube operator $\Gamma_{\mu \nu \lambda}$. Since the action is written by $\tilde{U}_{\mu \nu}$ and $\Gamma_{\mu \nu \lambda}$, it is manifestly invariant under the two-form gauge transformation.

The expectation value of an operator $\hat{O}$ is given by using the path integral as

$$
\langle\hat{O}\rangle=\frac{\int \mathcal{D} A_{\mu} \mathcal{D} B_{\mu \nu} e^{-S_{\text {lat }}} O}{\int \mathcal{D} A_{\mu} \mathcal{D} B_{\mu \nu} e^{-S_{\text {lat }}}} .
$$

Since $e^{-S_{\text {lat }}}$ is real and positive, we can compute $\langle\hat{O}\rangle$ on the basis of the standard techniques of Monte Carlo sampling. In this work, we generated gauge configurations by the heat bath algorithm. We also adopted the over-relaxation method [20] between the heat bath updates.

\section{INTERSTRING POTENTIAL}

Before discussing the interstring potential in the twoform lattice gauge theory, let us recall the interparticle potential in one-form lattice gauge theory. Considering a rectangle with length $r$ and width $T$ in the $\mu-\nu$ plane, we can construct a gauge-invariant observable as

$W_{L}(r, T)=\left\langle U_{\nu}^{\dagger}(x, T) U_{\mu}^{\dagger}(x+T \hat{\nu}, r) U_{\nu}(x+r \hat{\mu}, T) U_{\mu}(x, r)\right\rangle$

with

$$
U_{\mu}(x, r)=\Pi_{l=0}^{r-1} U_{\mu}(x+l \hat{\mu}) .
$$

The schematic figure is shown in Fig. 1. This is the socalled Wilson loop. The Wilson loop corresponds to the world lines of an infinitely heavy particle and antiparticle. The ground state energy of the particle-antiparticle pair, i.e., the interparticle potential, is obtained by

$$
a V_{q}(r)=\lim _{T \rightarrow \infty} \log \frac{W_{L}(r, T)}{W_{L}(r, T+1)} .
$$

The area law of the Wilson loop gives the linear confining potential between the particles. We remark here that this Wilson loop is always zero in our two-form lattice gauge theory because it is not gauge invariant under the two-form gauge transformation. In terms of the Abelian Higgs model, this implies the gauge dependence of magnetic monopoles.

This potential calculation can be generalized to the twoform lattice gauge theory. Considering a cuboid with the length $r$, width $L$, and height $T$ in the $\mu-\nu-\lambda$ space, we can construct a gauge-invariant observable as
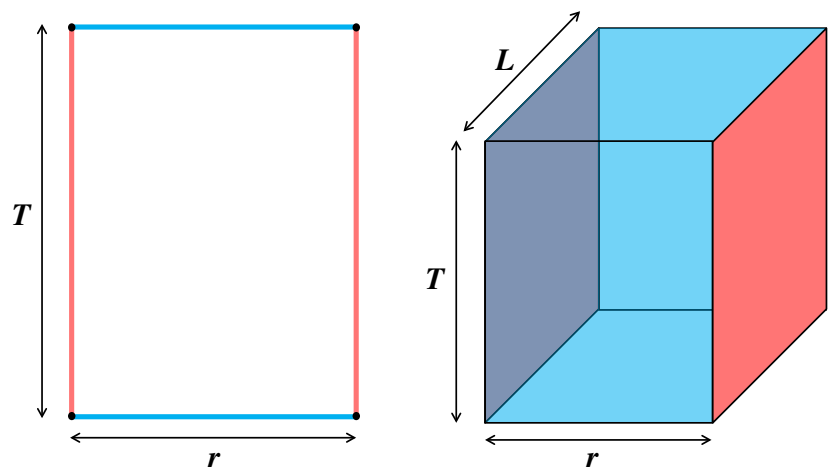

FIG. 1. Wilson loop (left) and Wilson surface (right). In the Wilson loop, the two red lines correspond to the trajectories of a particle and an antiparticle. In the Wilson surface, the two red surfaces correspond to the trajectories of a vortex string and an antivortex string. The blue lines and surfaces connect these trajectories to make the operators gauge invariant. 


$$
\begin{aligned}
& W_{S}(r, L, T) \\
&=\left\langle\Gamma_{\lambda \mu}^{\dagger}(x+L \hat{\nu}, T, r) \Gamma_{\nu \lambda}^{\dagger}(x+r \hat{\mu}, L, T) \Gamma_{\mu \nu}^{\dagger}(x+T \hat{\lambda}, r, L)\right. \\
&\left.\times \Gamma_{\lambda \mu}(x, T, r) \Gamma_{\nu \lambda}(x, L, T) \Gamma_{\mu \nu}(x, r, L)\right\rangle
\end{aligned}
$$

with

$$
\Gamma_{\mu \nu}(x, r, L)=\Pi_{l=0}^{r-1} \Pi_{m=0}^{L-1} \Gamma_{\mu \nu}(x+l \hat{\mu}+m \hat{\nu}),
$$

as shown in Fig. 1. In the limit of $L \rightarrow \infty$, this Wilson surface corresponds to the world sheets of an infinitely heavy and infinitely long string and antistring. The ground state energy is obtained by

$a V_{S}(r)=\lim _{T, L \rightarrow \infty} \log \frac{W_{S}(r, L, T+1) W_{S}(r, L+1, T)}{W_{S}(r, L, T) W_{S}(r, L+1, T+1)}$.

This is the interstring potential per unit length. The volume law of the Wilson surface gives the linear confining potential between the strings.

We computed the Wilson surface in the Monte Carlo simulation. The lattice volume is $V=16^{4}$. The APE smearing was employed to compute the interstring potential efficiently [21]. The result is shown in Fig. 2. At small $\kappa$, we clearly see the linearly rising potential. This is interpreted as the confinement between a string and antistring. We call it "string confinement" to distinguish it from the ordinary confinement between point particles. The linear potential disappears as $\kappa$ increases, which means "string deconfinement." This result suggests a new type of the phase transition characterized by the (de-)confinement of extended objects. This is the main result of this paper.

The numerical calculation was done in finite interstring distance. Does the linear confining potential persist in infinite distance? To answer this question, let us introduce another gauge-invariant observable

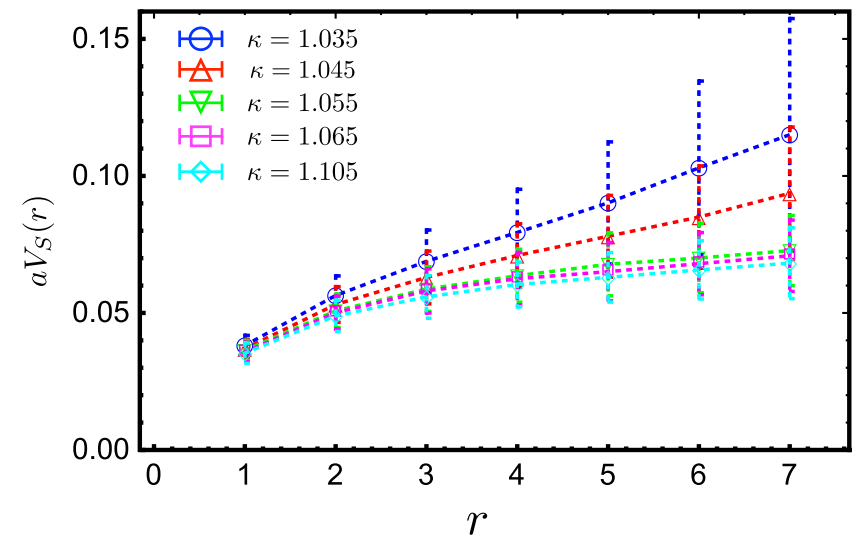

FIG. 2. Interstring potential with $\beta=4.1$, and various $\kappa$. The confining linear potential is clearly seen in the confinement phase at small $\kappa$, while it disappears in the deconfinement phase at large $\kappa$.

$$
W_{S}^{\prime}(r, L, T)=\left\langle\tilde{U}_{\nu \lambda}(x, L, T) \tilde{U}_{\nu \lambda}^{\dagger}(x+r \hat{\mu}, L, T)\right\rangle
$$

with

$$
\tilde{U}_{\mu \nu}(x, r, L)=\Pi_{l=0}^{r-1} \Pi_{m=0}^{L-1} \tilde{U}_{\mu \nu}(x+l \hat{\mu}+m \hat{\nu}) .
$$

The physical picture of this observable is the world sheets of a string and antistring attached with the one-form gauge field. Since the string and antistring are independently gauge invariant, they are not confined but weakly coupled. In this theory, $W_{S}(r, L, T)$ and $W_{S}^{\prime}(r, L, T)$ have the same quantum number. The two states, the confined and nonconfined states, are mixed. Since the ground state is the one with lower energy, the nonconfined state will be favored in large distance. Therefore, the corresponding potential will not be linear but constant. This is the same as the string breaking in quantum chromodynamics. The potential between a quark and antiquark is linear in short distance but constant in long distance. The confining string connecting the quark and antiquark is broken by dynamical quark-antiquark pair creation. In our case, the confining surface connecting a string and antistring will be broken by the one-form gauge field in the long-range limit. This should be called "surface breaking." The critical distance $r_{c}$ where the surface breaking takes place can be estimated by the energy balance relation $V_{S}\left(r_{c}\right)=2 M$. Here, $M$ is the mass of one gauge-invariant string defined by $\lim _{T, L \rightarrow \infty}\left\langle\tilde{U}_{\nu \lambda}(x, L, T)\right\rangle \propto \exp (-M L T)$. In this simulation, the typical values are $2 a M \simeq 0.38$ at $\beta=4.1$ and $\kappa=1.035,2 a M \simeq 0.14$ at $\beta=4.1$ and $\kappa=1.065$, and $2 a M \simeq 0.13$ at $\beta=4.1$ and $\kappa=1.105$. We see that the data in Fig. 2 are below the critical distance. The direct simulation of the surface breaking would be an interesting future work. In principle, both $W_{S}(r, L, T)$ and $W_{S}^{\prime}(r, L, T)$ can give the same correct result in the limit $T, L \rightarrow \infty$. In practice, however, special treatment is necessary for technical reasons [22].

\section{PHASE DIAGRAM}

We draw the phase diagram of this theory in the parameter space of $\kappa$ and $\beta$. We calculated the susceptibility

$$
\chi_{\kappa}=\frac{1}{V}\left\langle\left(\frac{\partial S_{\text {lat }}}{\partial \kappa}-\left\langle\frac{\partial S_{\text {lat }}}{\partial \kappa}\right\rangle\right)^{2}\right\rangle
$$

to determine the position and the order of the phase transition. Parallel tempering was employed to compute $\chi_{\kappa}$ [23]. As examples, we show the volume dependence of $\chi_{\kappa}$ at $\beta=4.0$ and 4.1 in Fig 3. At $\beta=4.1$, we observed the double peak structure implying metastable states and the volume growth of the susceptibility $\chi_{\kappa} \propto V$. Both are strong evidence of the first-order phase transition.

The obtained phase diagram is shown in Fig. 4. The finite-size scaling analysis was done for three lattice 

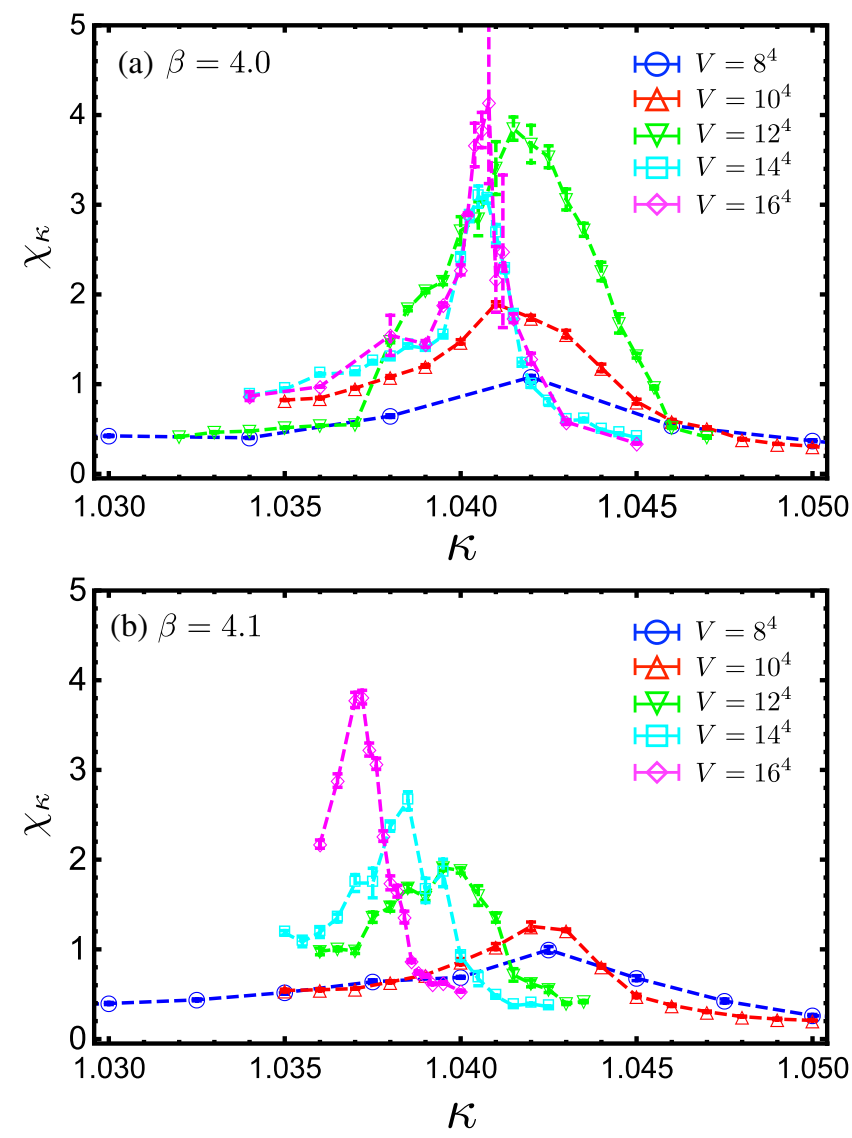

FIG. 3. Volume dependence of the susceptibility $\chi_{\kappa}$ : (a) $\beta=4.0$ below the critical point and (b) $\beta=4.1$ above the critical point. The last three volumes are used for the finite-size scaling analysis to determine the critical $\kappa$ in the infinite volume limit.

volumes $V=12^{4}, 14^{4}$, and $16^{4}$. There are two phases: the string confinement phase and the string deconfinement phase. In small $\beta$, the two phases are smoothly connected by a crossover. In large $\beta$, the two phases are separated by a first-order phase transition. In the limit of $\beta \rightarrow \infty$, the lattice action (3) reduces to the conventional one-form

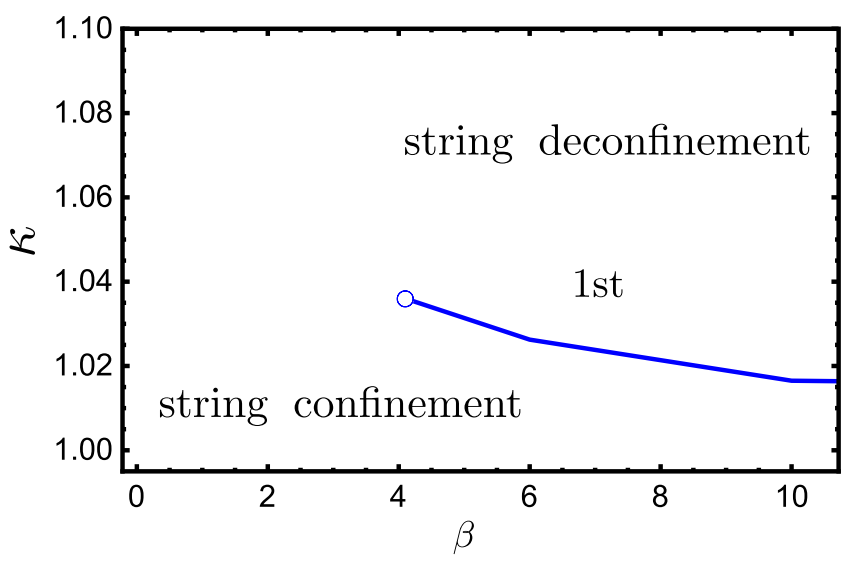

FIG. 4. Phase diagram of the two-form lattice gauge theory. The blue curve is the first-order phase-transition line.

compact $\mathrm{U}(1)$ gauge action. There must be a first-order phase transition in this limit. This is consistent with our observation. The first-order phase-transition line ends at a critical point. The position of the critical point was estimated as $\kappa_{c} \simeq 1.036$ and $\beta_{c} \simeq 4.1$.

This is the phase diagram in the case of the unit charge. The theory can be generalized to the charge $N$ representation by replacing $\tilde{U}_{\mu \nu}=U_{\mu \nu} \Gamma_{\mu \nu} \rightarrow U_{\mu \nu}\left(\Gamma_{\mu \nu}\right)^{N}$ in the lattice action (3). This is analogous to the phase diagram with the charge- $N$ Higgs field [24]. The phase diagram for the charge $N>1$ would be more interesting because the theory has $Z_{N}$ topological order.

\section{ACKNOWLEDGMENTS}

The authors thank Tin Sulejmanpasic for pointing out the possibility of surface breaking. T.H. thanks Takumi Doi, Sinya Gongyo, and Yuta Kikuchi for helpful comments. T. H. was supported by RIKEN special postdoctoral program. A. Y. was supported by JSPS KAKENHI Grant No. 19K03841.
[1] B. Rosenstein and D. Li, Rev. Mod. Phys. 82, 109 (2010).

[2] M. Tsubota, K. Kasamatsu, and M. Kobayashi, Novel Superfluids (Oxford University Press, Oxford, 2010), Vol. 1, Chap. 3, p. 156.

[3] M. Eto, Y. Hirono, M. Nitta, and S. Yasui, Prog. Theor. Exp. Phys. 2014, 12D01 (2014).

[4] M. B. Hindmarsh and T. W. B. Kibble, Rep. Prog. Phys. 58, 477 (1995).

[5] P. Orland, Nucl. Phys. B205, 107 (1982).

[6] R. B. Pearson, Phys. Rev. D 26, 2013 (1982).
[7] J. Frohlich and T. Spencer, Commun. Math. Phys. 83, 411 (1982).

[8] R. I. Nepomechie, Nucl. Phys. B212, 301 (1983).

[9] P. Orland, Phys. Lett. 122B, 78 (1983).

[10] C. Omero, P. A. Marchetti, and A. Maritan, J. Phys. A 16, 1465 (1983).

[11] A. E. Lipstein and R. A. Reid-Edwards, J. High Energy Phys. 09 (2014) 034.

[12] D. A. Johnston, Phys. Rev. D 90, 107701 (2014).

[13] M. Baig and J. Clua, Phys. Rev. D 57, 3902 (1998). 
[14] K. Kajantie, M. Laine, T. Neuhaus, A. Rajantie, and K. Rummukainen, Phys. Lett. B 482, 114 (2000).

[15] S. Wenzel, E. Bittner, W. Janke, and A. M. J. Schakel, Nucl. Phys. B793, 344 (2008).

[16] R. MacKenzie, F. Nebia-Rahal, and M. B. Paranjape, Phys. Rev. D 81, 114505 (2010).

[17] T. Hayata and A. Yamamoto, Phys. Rev. A 92, 043628 (2015).

[18] A. Yamamoto, Prog. Theor. Exp. Phys. 2018, 103 B03 (2018).
[19] F. Quevedo and C. A. Trugenberger, Nucl. Phys. B501, 143 (1997).

[20] M. Creutz, Phys. Rev. D 36, 515 (1987).

[21] M. Albanese et al., Phys. Lett. B 192, 163 (1987).

[22] G. S. Bali, H. Neff, T. Duessel, T. Lippert, and K. Schilling, Phys. Rev. D 71, 114513 (2005)

[23] K. Hukushima and K. Nemoto, J. Phys. Soc. Jpn. 65, 1604 (1996).

[24] E. H. Fradkin and S. H. Shenker, Phys. Rev. D 19, 3682 (1979). 